\title{
Síndrome ocular isquêmica simulando retinopatia diabética unilateral: Relato de caso e revisão da literatura
}

\author{
Ischemic ocular syndrome simulating unilateral diabetic \\ retinopathy: case report and literature review
}

Frederico Castelo Moura', Vitor Kazuo Lotto Takahashi², Eduardo Minelli ${ }^{3}$

\begin{abstract}
RESUMO
A síndrome ocular isquêmica (SOI) ocorre devido à hipoperfusão ocular crônica secundária à obstrução da artéria carótida. O quadro clínico inclui, entre outros, retinopatia proliferativa similar a retinopatia diabética. A SOI deve ser considerada principalmente nas retinopatias proliferativas unilaterais ou muito assimétricas e nos casos refratários ao tratamento por fotocoagulação. A indicação da endarterectomia nos pacientes com SOI isolada não é bem definida. Este trabalho relata uma paciente com SOI simulando retinopatia diabética proliferativa unilateral e tratada por endarterectomia.

Descritores: Estenose das carótidas; Artéria carótida interna; Endarterectomia das carótidas; Retinopatia diabética; Isquemia; Síndrome; Relatos de caso
\end{abstract}

\begin{abstract}
Ischemic ocular syndrome (IOS) is caused by stenosis of carotid artery. Proliferative retinopathy is one of the signs and can lead to misdiagnosis with diabetic retinopathy. IOS must be considered in case of proliferative retinopathy asymmetric and refractory to laser treatment. Carotid endarterectomy in patients with IOS with no neurologic manifestations remains controversy. This paper reported a patient with IOS simulating diabetic retinopathy and treated with endarterectomy.

Keywords: Carotid stenosis; Carotid artery, internal; Endarterectomy, carotid; Diabetic retinopathy; Ischemia; Syndrome; Case reports
\end{abstract}

\footnotetext{
${ }^{1}$ Universidade de São Paulo (USP) - São Paulo (SP), Brasil;

${ }^{2}$ Centro Universitário Lusíada (UNILUS) - Santos (SP), Brasil;

${ }^{3}$ Clínica de Oftalmologia Especializada (CLOE) - São Paulo - (SP), Brasil

Trabalho realizado na Clínica de Oftalmologia Especializada (CLOE) - São Paulo (SP), Brasil.

Os autores declaram inexistir conflitos de interesse

Recebido para publicação em 24/1/2011 - Aceito para publicação em 1/6/2011
} 


\section{INTRODUÇÃO}

S índrome Ocular Isquêmica (SOI) ocorre devido à hipoperfusão ocular crônica causada por estenose acima de $90 \%$ da artéria carótida interna ou carótida comum. ${ }^{(1)}$ Os sintomas da SOI podem incluir perda visual súbita, gradual ou transitória e dor ocular ou orbital. ${ }^{(2)}$ Os sinais clínicos do segmento anterior são uveíte e neovascularização de íris, e do segmento posterior são dilatação de vasos retinianos, hemorragia intrarretiniana extensa e proliferação neovascular ${ }^{(3)}$.

Diabetes mellitus (DM) é observada em mais da metade dos pacientes com $\mathrm{SOI}^{(3)}$ e por isso a retinopatia diabética (RD) é um dos principais diagnósticos diferenciais. $^{(4)} \mathrm{O}$ objetivo deste relato é apresentar uma paciente com SOI secundária à oclusão carotídea simulando RD proliferativa assimétrica. A indicação de endartarectomia de carótida na SOI também é discutida.

\section{Relato de caso}

Mulher, de 55 anos com queixa de episódios de perda súbita de visão, de resolução rápida associada à escotoma no olho esquerdo (OE) nos últimos 2,5 anos. Como antecedentes patológicos, apresentava DM insulino-dependente, hipertensão arterial sistêmica (HAS) controlada e tabagismo.

Ao exame oftalmológico, apresentou acuidade visual (AV) de 1,0 em ambos os olhos (AO), anisocoria (midríase à esquerda) com reflexos direto e consensual diminuídos à esquerda e ausência de rubeosis em AO. À tonometria de aplanação evidenciou $18 \mathrm{mmHg}$ em AO. À oftalmoscopia indireta apresentou raras drusas no polo posterior do olho direito (OD) e hemorragias retinianas nos quatro quadrantes do $\mathrm{OE}$. $\mathrm{O}$ exame de angiofluoresceinografia(AF) revelou micro-hemorragias e microaneurismas nos quatro quadrantes do $\mathrm{OE}$, sem sinal de edema macular e neovasos de retina (Figura 1A). A hipótese diagnóstica inicial foi RD não-proliferativa.

Após dois meses, a paciente evoluiu com perda visual progressiva do $\mathrm{OE}(\mathrm{AV}=0,3)$. $\mathrm{O}$ exame de AF evidenciou áreas de má perfusão capilar, neovasos de papila e edema macular difuso, além das micro-hemorragias e microaneurismas no OE (Figura 1B). Panfotocoagulação retiniana foi realizada neste olho para o tratamento da retinopatia proliferativa.

Apesar do tratamento, o quadro oftalmológico evoluiu com persistência dos neovasos e do edema macular do OE (Figura 1C), e o olho direito permanecia sem sinais de RD. Diante deste quadro, foi solicitada ultrassonografia com Doppler das artérias carótidas que diagnosticou estenose da artéria carótida interna (ACI) > $90 \%$, confirmada pela angiografia arterial (Figura 2). A paciente foi encaminhada ao cirurgião vascular que indicou endarterectomia da ACI à esquerda. Após três meses da cirurgia vascular, a paciente retorna para nova avaliação com acuidade visual 1,0 em AO, sem sintomas visuais. $\mathrm{O}$ exame de AF revelou involução dos neovasos de papila e melhora das áreas de microangiopatia, de hipoperfusão capilar e do edema macular difuso (Figura 1D).

O seguimento de 30 meses após a endarterectomia não evidenciou recidiva da SOI, sinais de ataque isquêmico transitório (AIT) ou acidente vascular cerebral (AVC).

\section{Discussão}

A etiologia mais comum de SOI é a doença aterosclerótica unilateral ou bilateral da artéria carótida interna ou da bifurcação da artéria carótida comum ${ }^{(5)}$. Estima-se que aproximadamente $5 \%$ dos pacientes com estenose de artéria carótida apresentam SOI. Essa síndrome acomete mais indivíduos masculinos do que femininos, na taxa de 2:1, de faixa etária entre 50 e 80 anos. A taxa de mortalidade de pacientes com SOI em cinco anos é de $40 \%$, sendo a doença cardíaca a principal causa de morte, seguida de AVC e câncer ${ }^{(6)}$. Pacientes com HAS representam mais de dois terços dos pacientes com SOI, e com DM é encontrada em mais de metade dos casos de SOI. ${ }^{(6)}$ Além de DM e HAS, outros fatores de risco como tabagismo, hiperlipidemia, AIT e AVC prévio estão associados à $\mathrm{SOI}^{(7)}$.

A perda visual é o sintoma ocular mais encontrado na SOI presente em $70-90 \%$ dos pacientes, e pode atingir AV 0,3 ou pior em dois terços dos acometidos ${ }^{(5)}$. Comumente, a perda visual ocorre de forma progressiva em um período de semanas a meses, embora possa se apresentar de forma súbita associado à oclusão de artéria central da retina. Ao exame ocular, a SOI é caracterizada por manifestações no segmento anterior como incluindo dilatação das veias episclerais, edema corneano, células e "flare" na câmara anterior, pupila em média midríase e pouco reagente, catarata e neovascularização de íris e glaucoma neovascular. As manifestações do segmento posterior incluem dilatação venosa, hemorragias intrarretinianas em média perife-
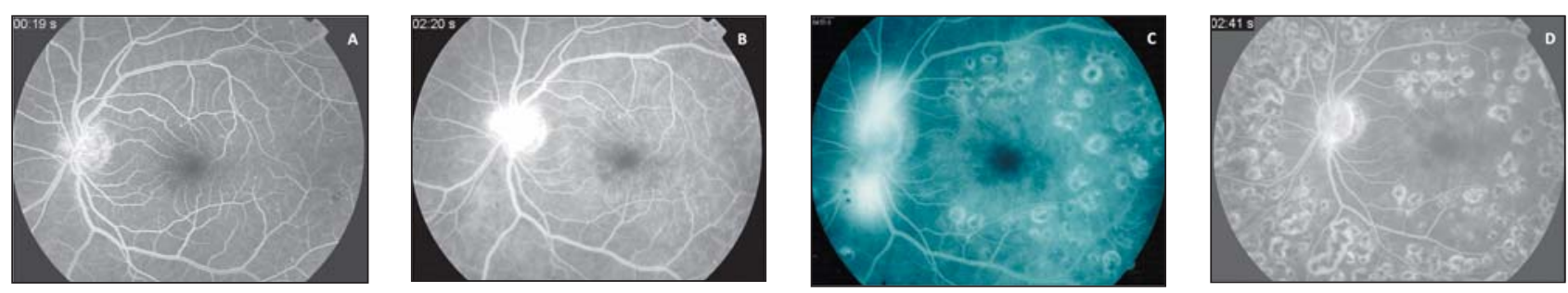

Figura 1: Exame de angiofluoresceinografia mostrando a evolução da síndrome ocular isquêmica; (A) fase pré-proliferativa evidenciando microaneurismas e micro-hemorragias; (B) fase proliferativa evidenciando extravasamento do contraste do disco óptico e edema macular difuso; (C) persistência dos achados da retinopatia proliferativa após fotocoagulação a laser; (D) resolução dos neovasos após endarterectomia da carótida 

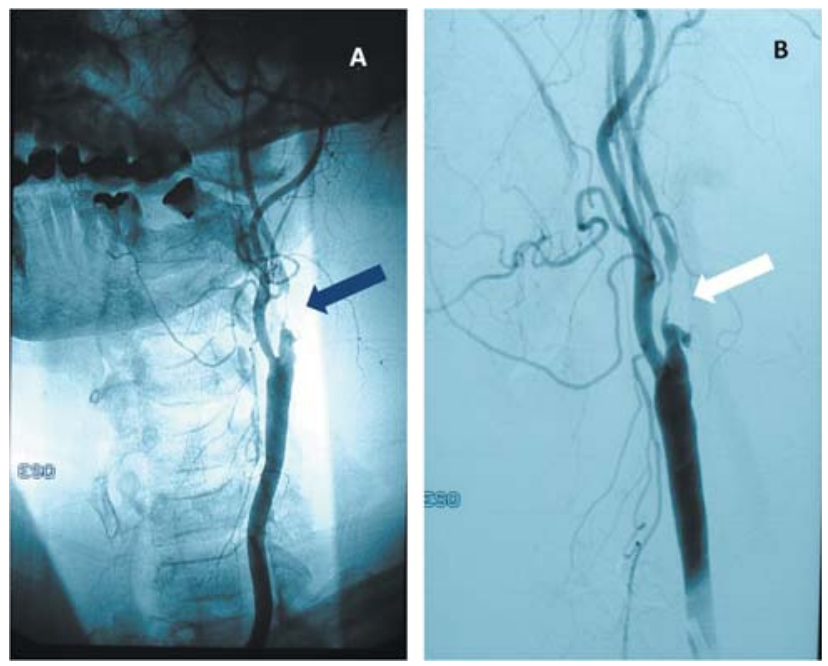

Figura 2: Exame de arteriografia convencional (A) e com supressão óssea digital (B), evidenciando a estenose suboclusiva da artéria carótida interna esquerda (setas).

ria, microaneurismas, neovascularização do disco e/ou retina, estreitamento arteriolar, tortuosidade vascular e edema macular ${ }^{(8)}$. Nosso paciente apresentou vários sintomas característicos da SOI, alem de fatores de risco como DM e HAS e tabagismo.

Embora clinicamente bem caracterizada, a SOI ainda oferece dificuldade diagnóstica devido à sua diversidade de apresentação e similaridade com afecções mais frequentes como a $\mathrm{RD}^{(4,9)}$. Essa patologia se apresenta inicialmente com microaneurismas retinianos assintomáticos, às vezes associados à edema macular e hemorragias. Devido a essas semelhanças com o quadro de SOI, a hipótese diagnóstica inicial para nossa paciente foi RD não-proliferativa ${ }^{(10)}$. $\mathrm{O}$ aumento da assimetria entre as manifestações oculares e a evolução desfavorável do quadro a despeito do tratamento sinalizaram uma etiologia diferente da RD confirmada pelo estudo angiográfico das carótidas.

Endarterectomia da carótida (EC) é um procedimento realizado em pacientes com estenose carotídea grave $(>70 \%)$ para prevenção de AVC com evidência baseada em estudos multicêntricos randomizados ${ }^{(11,12)}$. A eficácia da EC para prevenção de AVC nos pacientes com SOI com manifestações exclusivamente oculares foi investigada por vários autores e ainda se mantém controversa. Recentemente, Wolintz ${ }^{(13)}$ mostrou que entre as quatro condições oculares tais como: amaurose fugaz, SOI, oclusão de artéria central da retina e placa de Hollenhorst assintomática, associadas à estenose carotídea, apenas a amaurose fugaz associada à AIT ou AVC prévio, claudicação intermitente, estenose entre $80-94 \%$ e idade $>75$ mostrou benefício para o prognóstico ocular e cerebral após a EC. Para a SOI, o benefício não é bem definido porque as evidências são baseadas principalmente em casos isolados com documentação inapropriada e seguimento de curto prazo e apenas duas séries de casos publicados forneceram dados conclusivos. A primeira delas ${ }^{(14)}$ evidenciou melhora do quadro ocular na maioria dos pa- cientes e a outra ${ }^{(15)}$ mostrou que $60 \%$ dos pacientes submetidos à EC apresentavam perda visual após 12 meses. Não foram apresentados dados sobre prevenção de AVC nestas duas séries. Baseada na revisão citada ${ }^{(13)}$, nossa paciente não apresentava indicação para EC.

Concluindo, foi apresentada uma paciente com SOI por estenose de ACI, simulando retinopatia diabética proliferativa. Nós sugerimos que diante de retinopatia proliferativa muito assimétrica e/ou refratária ao tratamento seja realizada investigação do fluxo das artérias carótidas.

\section{REFERÊNCIAS}

1. Dugan JD Jr, Green WR. Ophthalmologic manifestations of carotid occlusive disease. Eye (Lond). 1991;5(Pt 2):226-38.

2. Mizener JB, Podhajsky P, Hayreh SS. Ocular ischemic syndrome. Ophthalmology. 1997;104(5):859-64.

3. Brown GC, Magargal LE. The ocular ischemic syndrome. Clini$\mathrm{cal}$, fluorescein angiographic and carotid angiographic features. Int Ophthalmol. 1988;11(4):239-51.

4. Garrido Neto TL, Halfeld R, Takahashi WY. Síndrome ocular isquêmica: relato de caso. Arq Bras Oftalmol. 1998;61:238-41.

5. Kahn M, Green WR, Knox DL, Miller NR. Ocular features of carotid occlusive disease. Retina. 1986;6(4):239-52.

6. Sivalingam A, Brown GC, Magargal LE, Menduke H. The ocular ischemic syndrome. II. Mortality and systemic morbidity. Int Ophthalmol. 1989;13(3):187-91.

7. Ino-ue M, Azumi A, Kajiura-Tsukahara Y, Yamamoto M. Ocular ischemic syndrome in diabetic patients. Jpn J Ophthalmol. 1999;43(1):31-5.

8. Arcieri ES, Costa VP. Síndrome ocular isquêmica associada a glaucoma neovascular: relato de um caso. Arq Bras Oftalmol. 2001;64(3):255-7.

9. Foncea Beti N, Mateo I, Díaz La Calle V, Ruiz J, Gomez Beldarrain M, Garcia-Monco JC. The ocular ischemic syndrome. Clin Neurol Neurosurg. 2003;106(1):60-2.

10. Kollias AN, Ulbig MW. Diabetic retinopathy: Early diagnosis and effective treatment. Dtsch Arztebl Int. 2010;107(5):7583; quiz 84. Review.

11. Randomised trial of endarterectomy for recently symptomatic carotid stenosis: final results of the MRC European Carotid Surgery Trial (ECST). Lancet. 1998;351(9113):1379-87. Comment in: Lancet. 1998;351(9113):1372-3. Lancet. 1998;352(9122):1434. Lancet. 1998;352(9122):144. Lancet. 1998;352(9122):143.

12. Barnett HJ, Taylor DW, Eliasziw M, Fox AJ, Ferguson GG, Haynes RB, et al. Benefit of carotid endarterectomy in patients with symptomatic moderate or severe stenosis. North American Symptomatic Carotid Endarterectomy Trial Collaborators. N Engl J Med. 1998;339(20):1415-25. Comment in: N Engl J Med. 1999;340(15):1210; author reply 1211-2. N Engl J Med. 1999;340(15):1209; author reply 1210-1. N Engl J Med. 1998;339(20):1468-71. ACP J Club. 1999;130(2):33. N Engl J Med. 1999;340(15):1209-10; author reply 1211.

13. Wolintz RJ. Carotid endarterectomy for ophthalmic manifestations: is it ever indicated? J Neuroophthalmol. 2005;25(4):299302. Comment in: J Neuroophthalmol. 2005;25(4):259-61.

14. Rubin JR, McIntyre KM, Lukens MC, Plecha EJ, Bernhard VM. Carotid endarterectomy for chronic retinal ischemia. Surg Gynecol Obstet. 1990;171(6):497-501.

15. Sivalingam A, Brown GC, Magargal LE. The ocular ischemic syndrome. III. Visual prognosis and the effect of treatment. Int Ophthalmol. 1991;15(1):15-20.

\section{Autor correspondente:}

Frederico Castelo Moura

Rua Itapeva, $\mathrm{n}^{\circ} 240-4^{\circ}$ andar

CEP 01332-000 - São Paulo (SP), Brasil

Email: fredcastelo@terra.br 